\title{
Thermal fixing of 10,000 holograms in $\mathrm{LiNbO}_{3}: \mathrm{Fe}$
}

\author{
Xin An, Demetri Psaltis, and Geoffrey W. Burr
}

\begin{abstract}
We discuss thermal fixing as a solution to the volatility problem in holographic storage systems that use photorefractive materials such as $\mathrm{LiNbO}_{3}: \mathrm{Fe}$. We present a systematic study to characterize the effect of thermal fixing on the error performance of a large-scale holographic memory. We introduce a novel, to our knowledge, incremental fixing schedule to improve the overall system fixing efficiency. We thermally fixed 10,000 holograms in a $90^{\circ}$-geometry setup by using this new schedule. All the fixed holograms were retrieved with no errors. (C) 1999 Optical Society of America
\end{abstract}

OCIS codes: $090.0090,120.6810,210.4680$.

\section{Introduction}

Volume holographic memories that use photorefractive materials have attracted interest because of their potential in high-capacity storage and fast parallel readout. After the successful demonstration of the storage capability and high-fidelity retrieval of 5000 holograms in a $\mathrm{LiNbO}_{3}: \mathrm{Fe}$ crystal, ${ }^{1}$ significant progress was achieved toward the development of a practical memory system. ${ }^{2,3}$ The volatility of the stored data is a serious impediment to the practical realization of photorefractive holographic memories. The same charge excitation and transport mechanism responsible for the writing of holographic gratings also erases them on further illumination, leading to the loss of stored information during readout. Amodei and Staebler ${ }^{4}$ found that optically stored holograms in $\mathrm{LiNbO}_{3}: \mathrm{Fe}$ could be stabilized against readout by heating the crystal during or after the writing process to higher than $100{ }^{\circ} \mathrm{C}$, followed by illumination at room temperature. They proposed that, at high temperatures when ionic conductivity dominates electronic conductivity, the light-induced space-charge distribution is compensated by ionic space-charge of opposite sign. At room temperature the ionic conductivity is quite small compared with the photoconductivity of the electrons. Therefore

X. An (axin@sunoptics.caltech.edu) and D. Psaltis are with the Department of Electrical Engineering, Mail Stop 136-93, California Institute of Technology, Pasadena, California 91125. G. W. Burr is with the IBM Almaden Research Center, Department K18/ D2, 650 Harry Road, San Jose, California 95210.

Received 1 June 1998; revised manuscript received 3 September 1998.

0003-6935/99/020386-08\$15.00/0

(C) 1999 Optical Society of America the ionic replica of the holographic gratings remains nearly intact, and the stored information can be read out without erasure. Bollmann et al. ${ }^{5}$ suggested that $\mathrm{OH}^{-}$ions that enter the crystals during growth were likely to be responsible for thermal fixing. The presence of these ions can be detected by their optical absorption at approximately $2.87 \mu \mathrm{m}$. Vormann et $a l .{ }^{6}$ confirmed the importance of the $\mathrm{OH}^{-}$concentration in the fixing process but identified the associated $\mathrm{H}^{+}$protons as the mobile ions. This finding was further corroborated by Klauer ${ }^{7}$ in 1991.

Theoretical models have been developed to describe the formation of thermally fixed holograms in $\mathrm{LiNbO}_{3}$ (Refs. 8-11) on the basis of a set of equations similar to those first put forward by Kukhtarev. ${ }^{12}$ Thermal fixing can be carried out in one of two ways. The first consists of three steps-recording, heating, and revealing. During holographic recording, illumination by spatially varying intensity patterns causes photoexcited electrons to migrate under various transport mechanisms and become trapped at different acceptor sites, resulting in a spatially modulated charge concentration that is a replica of the exposing light pattern. This generates a spacecharge field that, in turn, gives rise to spatial modulation of the refractive index of the material by means of the electro-optic effect. After recording, the crystal is heated, and the protons become mobile. Fieldinduced proton migration compensates the spatially varying electronic charge distribution. In the revealing process the crystal is cooled down to room temperature and illuminated by uniform light. The electronic gratings are partially erased, leaving a net space-charge field that is stable against further optical erasure.

In the second scheme holograms are recorded in the crystal at elevated temperatures. Stabler et 


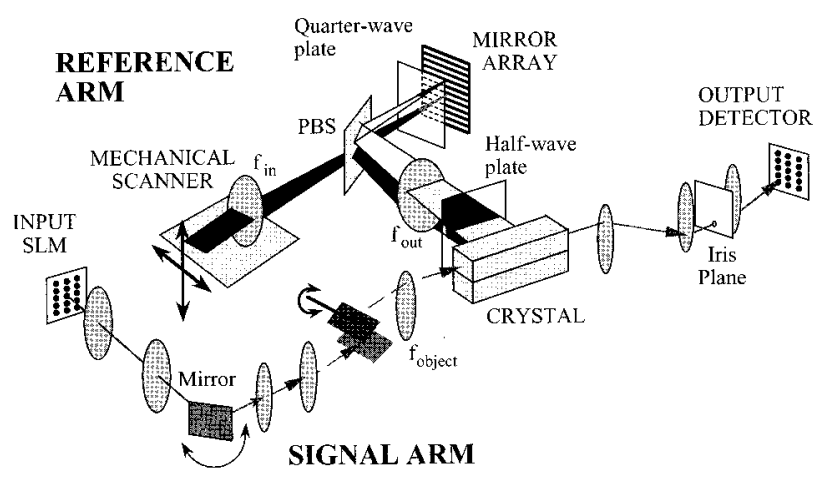

Fig. 1. Experimental setup for thermal fixing. SLM, spatial light modulator; PBS, polarizing beam splitter.

$a l .{ }^{13}$ reported that strong gratings with little noise can be recorded in this way. A disadvantage of this method is that, when the revealing process is carried out at lower temperatures, the fixed holograms suffer from distortion caused by thermal contraction and change of the refraction index of the material. ${ }^{14}$ Using both methods has demonstrated fixing of multiple holograms in $\mathrm{LiNbO}_{3}: \mathrm{Fe} .{ }^{13-15}$

In this paper we present an experimental characterization of the effects of the thermal-fixing process on system error performance. We show that, when more than 1000 holograms are stored, the loss in the signal-to-noise ratio (SNR) that results from the fixing process is a major obstacle to the practical realization of nonvolatile, large-scale holographic memories. We present and experimentally demonstrate a novel incremental fixing schedule that improves the overall fixing efficiency. We demonstrate experimentally that, for 10,000 fixed holograms, this new fixing method yields a SNR that is better than that obtained when we simply record and evaluate 10,000 unfixed holograms.

\section{Thermal Fixing of Multiple Holograms}

The fixing efficiency $\eta_{\text {fixing }}$ is the portion of the strength of the original hologram attained after the revealing process. Numbers ranging from as low as $\eta_{\text {fixing }} \approx 10^{-5}$ (Ref. 10) to as high as $70 \%$ (Ref. 16) have been reported. Most of the reported data are from single-hologram fixing experiments. We used an experimental setup with which we have demonstrated large-scale holographic storage ${ }^{17,18}$ to carry out a systematic study of the thermal-fixing efficiency and error performance that can be obtained when a large number of holograms are superimposed. A system diagram is shown in Fig. 1. This system stores as many as 160,000 holograms by use of angle, fractal, and spatial multiplexing. In the fixing experiments, we stored as many as 10,000 holograms at a single location. The crystal is $0.015 \%$ Fe-doped $\mathrm{LiNbO}_{3}$ with dimensions of $2 \mathrm{~cm} \times 1.5 \mathrm{~cm} \times 4 \mathrm{~cm}$. The $c$ axis was cut for $90^{\circ}$ geometry at $45^{\circ}$ to the illuminated faces. The crystal was placed $65 \mathrm{~mm}$ beyond the focal plane of the Fourier transform lens in the signal arm (a focal length of $f_{\text {obj }}=200 \mathrm{~mm}$ ) for

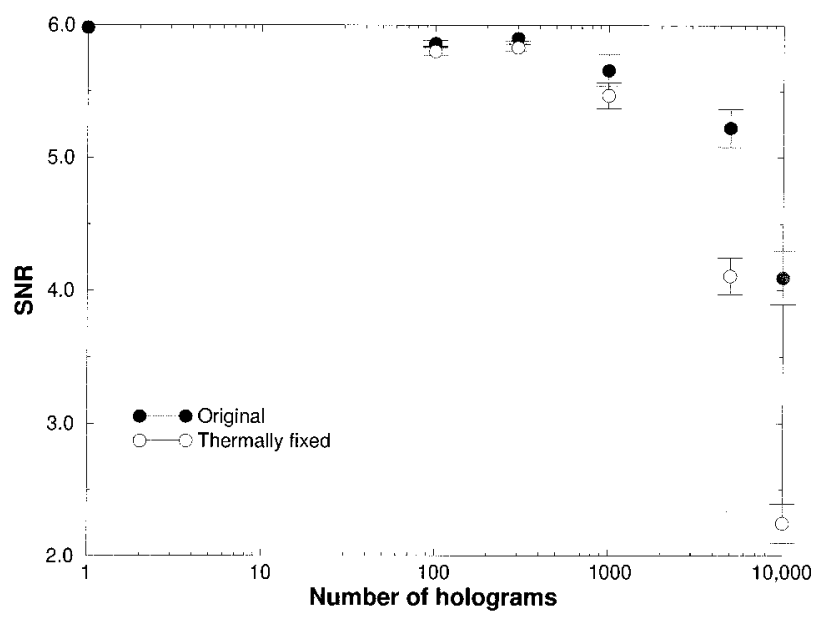

Fig. 2. SNR degradation with thermal fixing.

Fresnel plane recording. The images to be stored were all binary random-bit patterns. Each pattern consisted of $25 \times 80$ bits that were displayed on $480 \times$ 440 pixels on the input spatial light modulator. The reconstructions were captured by a cooled scientific CCD camera and transferred to a computer for data analysis. Data analysis involved registration of the $25 \times 80$ pixels and construction of the histograms of the OFF and the ON signals. Using these histograms as estimates of the probability-density functions of the binary signals, we define the SNR as

$$
\mathrm{SNR}=\frac{\mu_{1}-\mu_{0}}{\left(\sigma_{1}^{2}+\sigma_{0}^{2}\right)^{1 / 2}}
$$

where $\mu_{0}$ and $\mu_{1}$ are the means of the ofF and the oN signals, respectively, and $\sigma_{0}{ }^{2}$ and $\sigma_{1}^{2}$ are the corresponding variances.

We analyzed the SNR's of the reconstructions for the storage of one hologram and for the storage of $100,300,1000,5000$, and 10,000 original and thermally fixed holograms. In all cases the angular separation between neighboring holograms was at least 4 times the angle to the first null of the selectivity curve for each hologram. Five fractal rows ${ }^{19}$ were used for the recording of 5000 and 10,000 holograms, with 1000 and 2000 angularly multiplexed holograms in each row, respectively. The exposure schedule reported in Ref. 20 was followed to equalize the diffraction efficiencies of the recorded holograms. The exposure schedules for storing different numbers of holograms were selected so that the equalized diffraction efficiency was the same in all cases $\left(\approx 7 \times 10^{-9}\right)$. This equalization is accomplished by recording, for example, the 100 holograms as if they were the last 100 holograms in the 10,000-hologram sequence. The results are summarized in Fig. 2. The solid circles show the SNR's of the equalized holograms before fixing. We attribute the reduction in SNR as the total number of holograms increases to the development of fanning, interpixel-noise and other noise gratings over the long exposure sequence, and non- 
uniform erasure of the recorded holograms caused by absorption in the crystal.

We carried out thermal fixing by heating the crystal to $120{ }^{\circ} \mathrm{C}$. The crystal was allowed to cool and was then repositioned in the setup and illuminated with UV light to reveal the fixed holograms. We did not use the reference beam for the revealing process because, even with continuous scanning of the reference beam, a substantial increase in noise was observed. The average diffraction efficiency measured from all the experiments was $1.8 \times 10^{-9}$, leading to an average thermal-fixing efficiency of $26 \%$. Following Ref. 11 and assuming that the electronic gratings are fully compensated by the proton gratings after heating (a valid assumption as there was no measurable diffraction from the fixed holograms before revealing), we can write the fixing efficiency as

$$
\eta_{\text {fixing }}=\left(\frac{E_{\text {fixed }}}{E_{\text {original }}}\right)^{2}=\frac{\left(\frac{N_{D}-N_{A}}{N_{D}} E_{0 p h}\right)^{2}+E_{D}{ }^{2}}{\left(\frac{N_{D}-N_{A}}{N_{D}} E_{0 p h}\right)^{2}+\left(E_{D}+E_{q}\right)^{2}},
$$

where $E_{\text {original }}$ and $E_{\text {fixed }}$ are the amplitude of the space-charge field of the hologram before and after fixing, respectively, and $N_{D}$ and $N_{A}$ are the densities of the dopants and the ionized dopants, respectively. $E_{D}=k_{B} T K / q$ is the diffusion field, $E_{q}=q N_{A}\left(N_{D}-\right.$ $\left.N_{A}\right) / K \in N_{D}$ is the limiting space-charge field of the dopants, and the photovoltaic field is $E_{0 p h}=p \gamma_{R} N_{A} /$ $q \mu_{e} s$. For theoretical prediction of the thermalfixing efficiency, we used the parameters used in Ref. 21. The estimated fixing efficiency is $30 \%$, which is in good agreement with the $26 \%$ that was experimentally observed.

The SNR's of the thermally fixed holograms are represented by the open circles in Fig. 2. They remain close to those of the original holograms at the beginning, indicating faithful copying by means of thermal fixing. However, as the total number of holograms becomes larger, the SNR of the fixed holograms is significantly lower. We cannot attribute this drop to detector noise or scattering because the diffraction efficiency and the fixing efficiency were both independent of the number of holograms stored. Therefore the drop must be attributed to a noise source that is introduced by the fixing process and that becomes more prevalent when more (not weaker) holograms are stored. Detailed examination of the experimental data (Fig. 3) shows two effects: First, the mean and the variance of the ofF pixels rise as the number of holograms increases, whereas the mean of the on pixels stays constant. We attribute this outcome to a buildup of holographic noise, such as interpixel-grating noise. Second, the drop in the mean and the variance of the oN pixels arising from fixing is higher than the drop of the off pixels. This relation seems to indicate that the interpixel gratings experience a higher fixing efficiency. This conclusion is surprising because theory predicts [Eq. (2)]

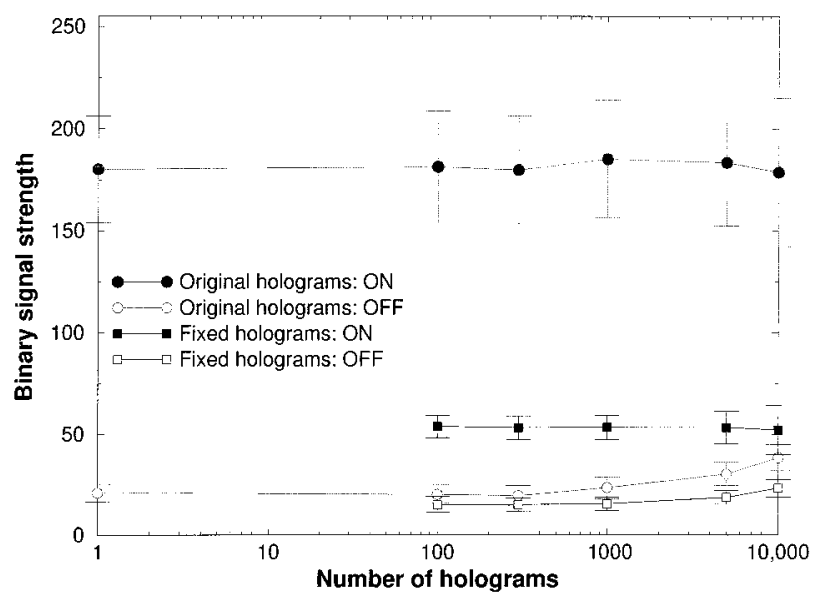

Fig. 3. Means and variances of the on and the OFF signals as functions of the number of holograms.

that the fixing efficiency should drop off at lower spatial frequencies. Independent measurements of the fixing efficiency as a function of spatial frequency have confirmed this unexpected trend. We do not yet have a satisfactory explanation for this observation.

To record and fix more than 1,000 holograms reliably, it is essential to improve the fixing efficiency. As shown in Eq. (2), the fixing efficiency is determined by the reduction-oxidation state of the crystal. In other words, the fixing efficiency is a function of the absorption coefficient, as indicated by curve (a) in Fig. 4. It shows that, as the absorption becomes weaker, the fixing efficiency becomes higher. However, when absorption is weaker, there are fewer donor sites $\left(\mathrm{Fe}^{2+}\right)$ available, and the $M / \#$ of the original system without fixing is smaller [curve (b) in Fig. 4]. As a result, there is an optimal absorption for the highest possible $M / \#$ after fixing, as shown by curve (c) in Fig. 4. The optimal absorption is $0.37 \mathrm{~cm}^{-1}$, and the crystal we used had an absorption of 0.55

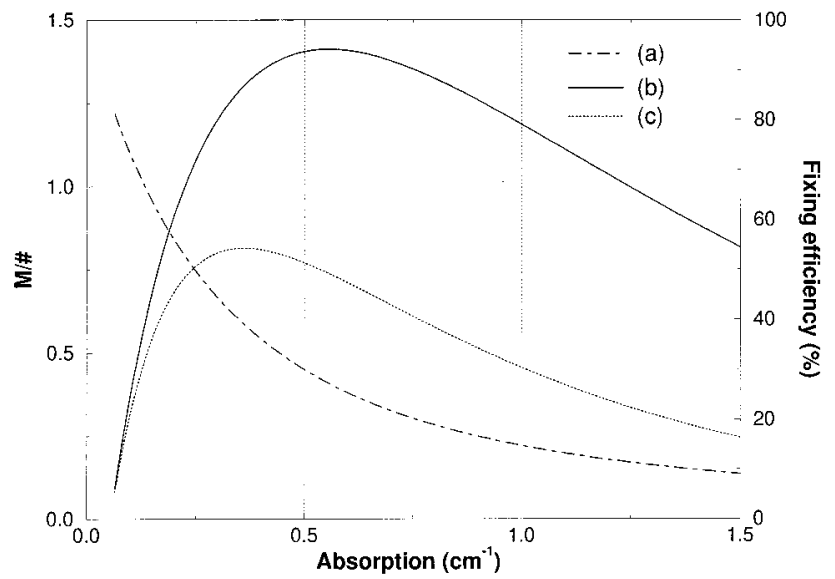

Fig. 4. $M / \#$ as a function of the absorption coefficient of the material: curve (a) represents thermal-fixing efficiency, curve (b) the $M / \#$ of the original system, and curve (c) the $M / \#$ of the thermally fixed system. 
$\mathrm{cm}^{-1}$. So there is little room for improvement in our experiment by means of changing the reductionoxidation state of the crystal.

\section{Incremental Fixing Schedule}

In the context of a large-scale holographic memory, thermal-fixing efficiency can be defined in two ways. First is the fixing efficiency for an individual hologram, as shown in Eq. (2). Second is the system thermalfixing efficiency, defined as the ratio of the final diffraction efficiency of multiple thermally fixed holograms, with $t_{1}$ as the recording time for the first hologram, to the diffraction efficiency of the same number of unfixed holograms starting with the same initial exposure time. Because of the dynamic nature of a memory system that uses $\mathrm{LiNbO}_{3}$ crystals, the recording of multiple holograms involves both writing and erasure behaviors. To store all the holograms with equal strength, we need to use a carefully chosen set of decreasing exposure times as the exposure schedule. The difference between the fixing efficiencies for an individual-hologram and a multiple-hologram system lies in the fact that the former's efficiency is determined solely by the material and the system parameters, whereas the latter's also depends on the writing and the erasure dynamics and thus can be improved by optimization of the exposure schedule.

The optical erasure of an unfixed hologram can be described as

$$
h(t)=h_{0} \exp \left(-t / \tau_{e}\right)
$$

where $h(t)$ is the electronic grating strength as a function of time. Equation (3) is simply an exponential decay with initial value $h_{0}$ and an erasure time constant $\tau_{e}$.

During erasure of a hologram after heating, when proton compensation takes place, although the spatially varying electronic-charge concentration is smoothed out by the migration of photoelectrons as in the normal case, it is still modulated by the existing proton grating formed during heating. Therefore the electronic grating does not drop exponentially to zero. Instead, it experiences a slower decay with a nonzero steady-state value (this explains why the thermal fixing efficiency is less than one). Its dynamics are given by

$$
h^{\prime}(t)=\left(1-\sqrt{\eta_{\text {fixing }}}\right) h_{0}{ }^{\prime}+\sqrt{\eta_{\text {fixing }}} h_{0}^{\prime} \exp \left(-t / \tau_{e}\right),
$$

where $h^{\prime}(t)$ is the electronic-grating strength as a function of time during erasure, $h_{0}{ }^{\prime}$ is its initial value immediately after heating, and $\eta_{\text {fixing }}$ is the thermalfixing efficiency defined in Eq. (2). Here we make the assumption that the imaginary term in the complex time constant is negligible. Therefore the decay time constant is also $\tau_{e}$. This assumption is valid provided that the total erasure time is not long compared with $\tau_{e}$.

The recording and the erasure behaviors in different cases are plotted in Fig. 5. This figure also provides a simple example of how the exposure schedule is made. First, hologram 1 is recorded for $t_{1}$. Be-

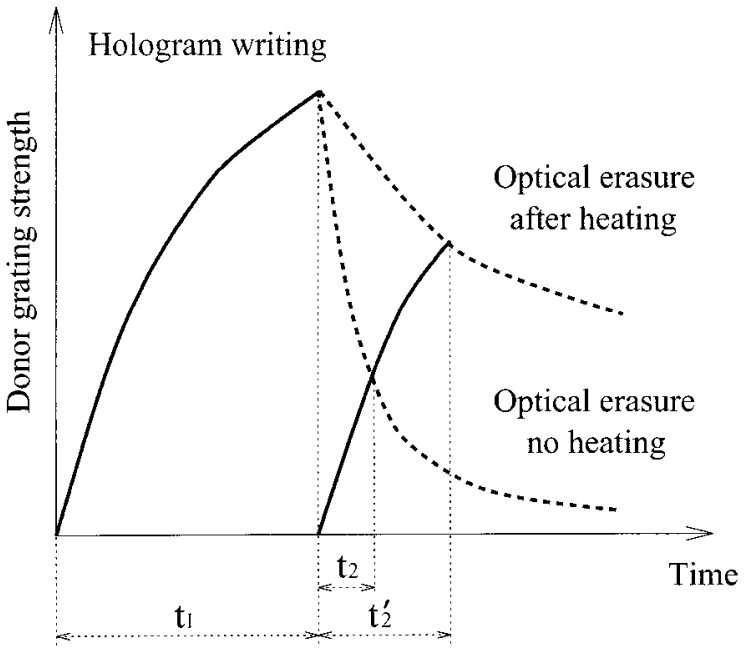

Fig. 5. Holographic recording and erasure under different conditions.

cause hologram 1 would decay on further exposure for hologram 2, the crystal can be exposed for only $t_{2}$ shorter than $t_{1}$ to make the strengths of the two holograms equal. However, if the crystal is heated after the hologram 1 is written, it decays much slower. As a result, hologram 2 can be recorded for $t_{2}{ }^{\prime}$, which is significantly longer and leads to an increase in the diffraction efficiency of both holograms. If the same treatment-recording followed by heating-is repeated for multiple times, higher overall diffraction efficiencies can be achieved at the end. However, in practice it is desirable to keep the number of heating treatments to a minimum. Therefore we break up the entire recording sequence of $M$ holograms into $M_{s}$ sets. Each set consists of an equal number of holograms, $M_{h}=M / M_{s}$. When the recording of a set of holograms is finished, the crystal is heated in an oven for a certain amount of time. After cooling and repositioning of the crystal, another set of $M_{h}$ holograms is recorded. The procedure is repeated until all $M$ holograms are recorded. Finally, the crystal is heated again and then illuminated with uniform light to reveal all the fixed holograms. We refer to this procedure as the incremental fixing schedule. Intuitively, when $M_{s}$ is smaller (the heating treatment is done less often), normal optical erasure becomes more dominant, and the gain in the final hologram strength is less. On the other hand, with smaller values of $M_{s}$, hologram recording takes less time and effort. As a result, in practice, there is always a trade-off.

In an exposure schedule for normal holographic recording, we achieve equal diffraction efficiency of all holograms by making the diffraction efficiency of the two neighboring holograms, the $m$ th and the $(m+1)$ th holograms, the same ${ }^{20}$ :

$$
\begin{array}{r}
h_{0}\left[1-\exp \left(-t_{m} / \tau_{e}\right)\right] \exp \left(-t_{m+1} / \tau_{e}\right) \\
=h_{0}\left[1-\exp \left(-t_{m+1} / \tau_{e}\right)\right],
\end{array}
$$


where $t_{m}$ and $t_{m+1}$ are the exposure times for the $m$ th and the $(m+1)$ th holograms, respectively. Typically, the exposure schedule is generated by use of a backward recursive algorithm, given the total number of holograms to be stored $M$, the erasure time constant $\tau_{e}$, and the exposure time for the last hologram $t_{M}$. Alternatively, an exposure schedule can be calculated by use of a forward method that starts with $t_{1}$ as the exposure time for the first hologram. The final diffraction efficiency of the system is then the efficiency of the decayed first hologram because of the exposures required for recording the rest $(M-1)$ of the holograms with equal strength. In a purely theoretical treatment in which $t_{1}$ can be infinitely long, so the first hologram is written to saturation, the diffraction efficiency approaches the upper limit imposed by the system's dynamic range, as described by the $M / \# .{ }^{20,21}$ For the practical implementation of a large-scale memory that favors a much shorter $t_{1}$ to avoid the buildup of holographic noise, we choose $t_{1}=$ $\tau_{e} / R_{1}\left(R_{1}>1\right)$ and use it in Eq. (5). The exposure time for the $m$ th hologram is then

$$
t_{m}=\frac{\tau_{e}}{R_{1}+m-1}
$$

where $m=1,2, \ldots, M$.

For designing the incremental fixing schedule, the only modification of Eq. (5) is that, instead of equalizing neighboring holograms, neighboring sets of holograms are equalized. In other words, the decreased diffraction efficiency of the $n$th set of holograms caused by optical erasure after heating is matched to that of the $(n+1)$ th set of holograms. During the recording of the holograms within a set, the optical erasure is the same as in the normal case. Therefore a normal exposure schedule is applied to the storage of each set of holograms. Given the number of holograms in a set $M_{h}$ and the erasure time constant $\tau_{e}$, only the recording time for the first hologram in the $n$th set, $t_{1}{ }^{n}$, is needed to specify the exposure schedule for the entire set. The values of $t_{1}{ }^{n}\left(n=1,2, \ldots, M_{s}\right)$ are calculated with

$$
\begin{gathered}
\left(1-\sqrt{\eta_{\text {fixing }}}\right) h_{0}{ }^{n}+\sqrt{\eta_{\text {mixing }}} h_{0}{ }^{n} \exp \left[-\sum_{i=1}^{M_{h}} t_{i}{ }^{n+1} / \tau_{e}\right]=h_{0}{ }^{n+1}, \\
h_{0}{ }^{n}=h_{\infty}\left[1-\exp \left(-t_{M_{h}}{ }^{n} / \tau_{e}\right)\right], \\
h_{0}{ }^{n+1}=h_{\infty}\left[1-\exp \left(-t_{M_{h}}{ }^{n+1} / \tau_{e}\right)\right],
\end{gathered}
$$

where $h_{0}{ }^{n}$ and $h_{0}{ }^{n+1}$ are the strengths of the $n$th and the $(n+1)$ th sets of holograms after recording, respectively. Correspondingly, $t_{M_{h}}{ }^{n}$ and $t_{M_{h}}{ }^{n+1}$ are the last exposure times for each set. The term $h_{\infty}$ is the saturation value. The left-hand side of Eq. (7) accounts for the decay of the $n$th set of holograms during the recording of the $(n+1)$ th set, which takes $\sum_{i=1}^{M_{h}}$ $t_{i}^{n+1}$. Each $t_{i}^{n+1}$ is simply derived from the normal exposure schedule starting with $t_{1}{ }^{n+1}$ [Eq. (6)].

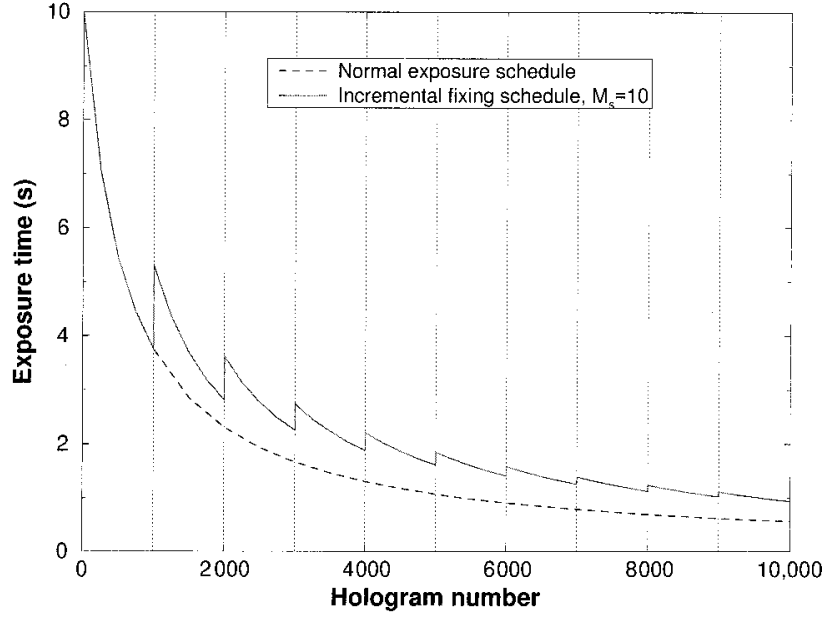

Fig. 6. Example of the incremental fixing schedule.

Combining Eqs. (6) and (7) yields the first exposure time $t_{1}{ }^{n}$ for the $n$th set as

$$
t_{1}^{n}=\frac{\tau_{e}}{R_{1}+\sqrt{\eta_{\text {fixing }}(n-1) M_{h}}},
$$

where $n=1,2, \ldots, M_{s}$ and $R_{1}=\tau_{e} / t_{1}$ corresponds to the choice of the first exposure time for the entire incremental fixing schedule. It should be noted that the exposure time of the first hologram in the $n$th set, $t_{1}{ }^{n}$, corresponds to that of the $\left[(n-1) M_{h}+1\right]$ th hologram in the entire exposure sequence. In the normal exposure schedule [Eq. (6)], the exposure time for this hologram would be

$$
\frac{\tau_{e}}{R_{1}+(n-1) M_{h}} .
$$

Comparing Eqs. (8) and (9) clearly shows that, because $\eta_{\text {fixing }}<1$, the exposure time of the same hologram is longer with the incremental fixing schedule.

Figure 6 shows an example in which the total number of holograms to be stored is $M=10,000$, the number of heating treatments is $M_{s}=10$, and the number of holograms in each set is $M_{h}=1000$. The erasure time constant is taken as $6000 \mathrm{~s}$, and the first exposure time is $t_{1}=10 \mathrm{~s}\left(R_{1}=600\right)$. Because the crystal is heated after the recording of each set of 1000 holograms, the exposure times at holograms $1001,2001, \ldots, 9001$ are increased, resulting in overall stronger hologram strength at the end of recording.

After the entire recording sequence is finished, the crystal is heated and then illuminated to reveal the fixed holograms. The diffraction efficiency of the fixed holograms is the product of the efficiency obtained at the end of the recording and the thermalfixing efficiency for an individual hologram. The ratio of this diffraction efficiency and that of the $M$ unfixed holograms is the system's fixing efficiency. It is plotted as a function of the number of heating treatments (the number of sets $M_{s}$ ) in Fig. 7. The 


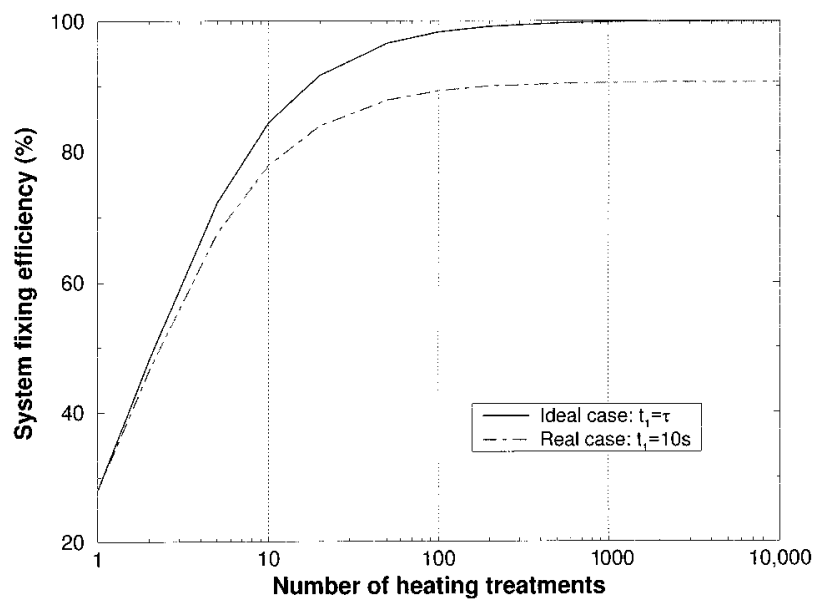

Fig. 7. System fixing efficiency as a function of the number of heating treatments.

total number of holograms to be stored $M$ is 10,000 , and the erasure time constant $\tau_{e}$ is taken as $6000 \mathrm{~s}$. In Fig. 7, curve (a) represents the practical case in which the recording is started with $t_{1}=10 \mathrm{~s}$. It shows that, as $M_{s}$ increases (the crystal is heated more often), the system's fixing efficiency becomes

higher. Curve (b) in Fig. 7 shows an ideal case in which $t_{1}$ can be infinitely long. It predicts that, as $M_{s}$ comes closer to $M$, the system's fixing efficiency approaches $100 \%$. In other words, at the limit where $M_{s}=M$ (heating is applied after the recording of every hologram), the gain in the exposure schedule from the incremental heating treatment compensates all losses that are due to thermal fixing and the maximum $M$ /\# of the memory system with incremental fixing is the same as that of the original volatile one.

\section{Thermal Fixing of 10,000 Holograms}

Using the same setup shown in Fig. 1, we recorded and thermally fixed 10,000 holograms by using an incremental fixing schedule. Five fractal rows were used for storage, with 2000 holograms stored in each. The vertical spacing between fractal rows was $2.4^{\circ}$, and the horizontal spacing between neighboring holograms was $0.007^{\circ}$, approximately 4 times the measured Bragg selectivity. To avoid overlapping that might occur because of imperfect repositioning, we set the angle spacing between two adjacent sets of holograms on the same fractal row to be 10 times wider than the hologram spacing.

In the incremental fixing schedule, 10,000 holo-
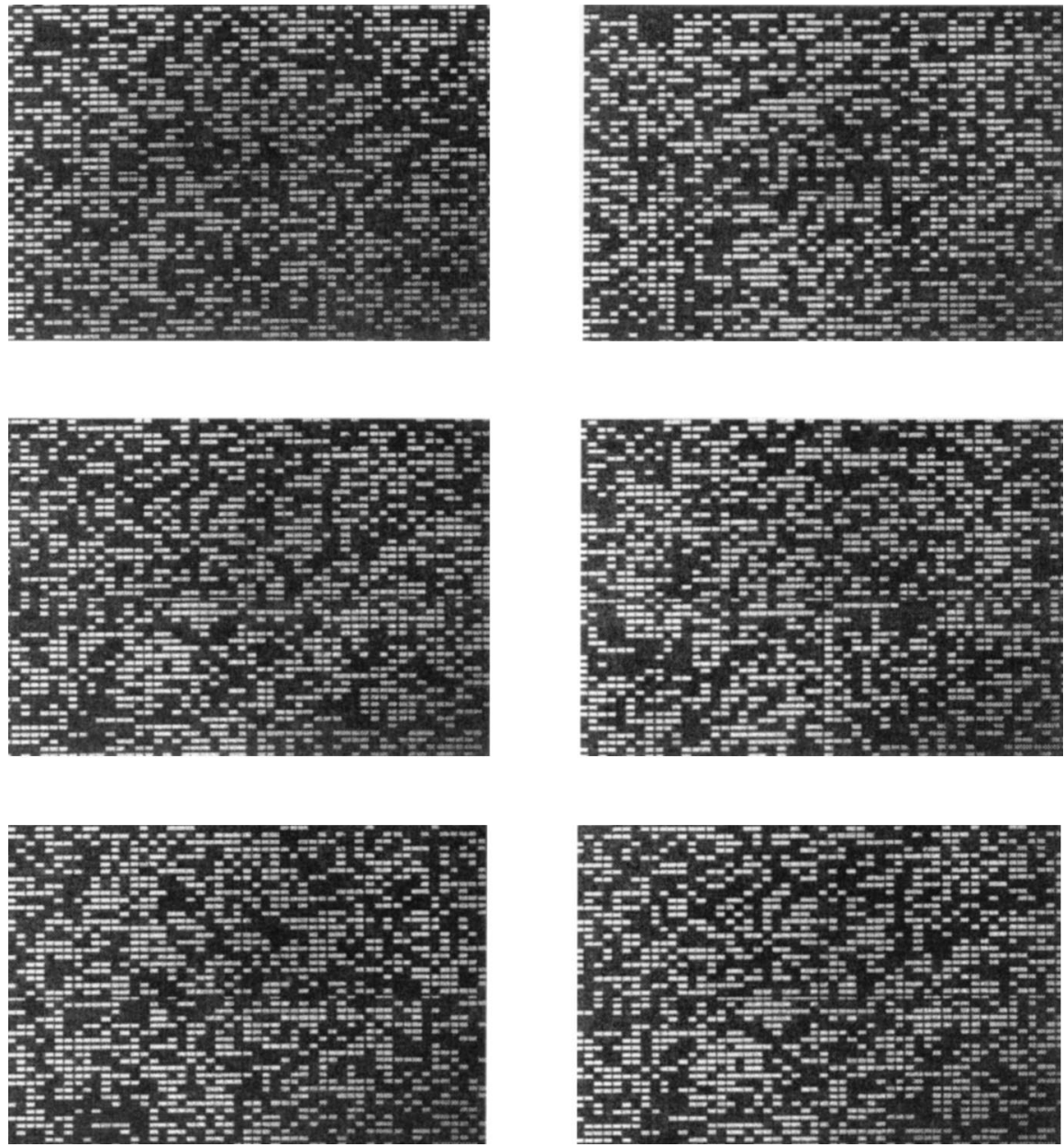

Fig. 8. Sample reconstructions from the 10,000 fixed holograms by use of the incremental fixing schedule. 

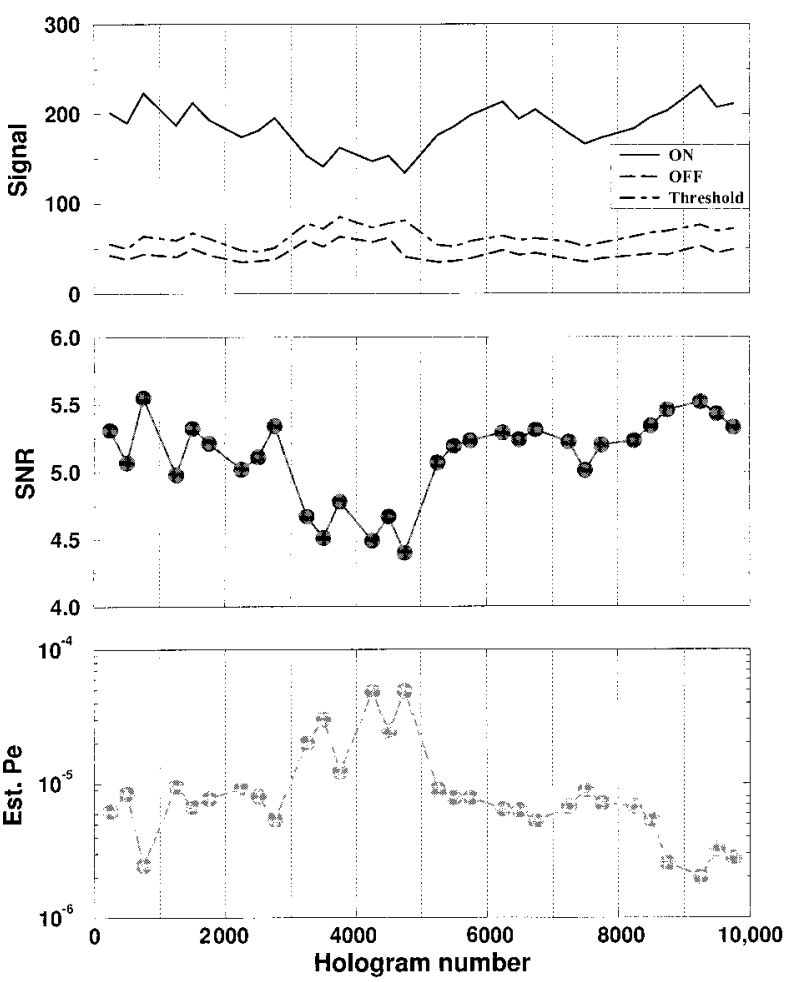

Fig. 9. Characteristics of the sample reconstructions from the 10,000 fixed holograms. Est. Pe., estimated probability of the error.

grams were divided into 10 sets $\left(M_{s}=10\right)$, two in each fractal row. The crystal was heated in an oven preheated to $120^{\circ} \mathrm{C}$ for $30 \mathrm{~min}$ after each set of $M_{h}=$ 1000 holograms were recorded. The erasure time constant $\tau_{e}$ was determined empirically to be $6000 \mathrm{~s}$. The first exposure time $t_{1}$ was chosen to be $10 \mathrm{~s}$. The exposure time for the 10,000 th hologram $t_{10,000}$ was calculated from Eqs. (6) and (8) to be $0.94 \mathrm{~s}$. This exposure time was nearly twice as long as the final exposure when the normal exposure schedule without thermal fixing was used. However, because of the loss during revealing $\left(1-\eta_{\text {fixing }} \approx 70 \%\right)$, the final system fixing efficiency is expected to be $78 \%$ (see Fig. 7). This value is more than twice the individual fixing efficiency, without the incremental schedule.

After 10,000 holograms were recorded and thermally fixed, 30 sample reconstructions were retrieved by a cooled scientific CCD camera for further data analysis. The samples were well distributed among the 10,000 holograms, and some are shown in Fig. 8. The same data-analysis procedure that was described in Section 3 was used to analyze error performance. The average diffraction efficiency was appoximately $4.6 \times 10^{-9}$. Compared with that of the 10,000 holograms without fixing $\left(\eta \approx 7 \times 10^{-9}\right)$, the incremental fixing schedule yielded a system fixing efficiency of roughly $66 \%$, close to the estimated value from Fig. 7 . All the sample reconstructions were retrieved without any measured errors. However, we observed nonuniformity, which could be attributed to imperfect repositioning, across the reconstructed images. The characteristics of these reconstructions are plot- ted in Fig. 9, including the means of the on and the OFF pixel regions, the optimal threshold, the SNR, and the estimated probability of error of the reconstructed holograms. It is very interesting to note that, even with the lower diffraction efficiency, the error performance of the 10,000 fixed holograms is comparable with or even slightly better than that of the unfixed holograms. ${ }^{18}$ This result is not surprising because the repeated heating reduces the electric field inside the crystal, resulting in less scattered noise. Similar effects have been observed by other researchers. ${ }^{13}$

\section{Conclusion}

We have conducted a series of experiments to characterize the effect of the thermal-fixing process on system error performance of a large-scale holographic memory that uses a $\mathrm{LiNbO}_{3}: \mathrm{Fe}$ crystal. The reduction in the SNR after thermal fixing is due to the low fixing efficiency. We have described a novel incremental fixing schedule that takes advantage of the dynamic nature of holographic recording to increase the diffraction efficiency of multiple thermally fixed holograms. We successfully fixed 10,000 holograms in our experiment by using this schedule, with greatly improved error performance.

This study was supported by the Rome Air Development Center and the U.S. Air Force Office of Scientific Research.

\section{References and Note}

1. F. H. Mok, "Angle-multiplexed storage of 5000 holograms in lithium niobate," Opt. Lett. 18, 915-917 (1991).

2. D. Psaltis and F. H. Mok, "Holographic memories," Sci. Am. 273,(5) 70-76 (1995).

3. J. F. Heanue, M. C. Bashaw, and L. Hesselink, "Volume holographic storage and retrieval of digital data," Science 265(5173), 749-752 (1994).

4. J. J. Amodei and D. L. Staebler, "Holographic pattern fixing in electro-optic crystals," Appl. Phys. Lett. 18, 540-542 (1971).

5. W. Bollmann and H. J. Stöhr, "Incorporation and mobility of $\mathrm{OH}^{-}$ions in $\mathrm{LiNbO}_{3}$ crystals," Phys. Status Solidi A 39, 477484 (1977).

6. H. Vormann, G. Weber, S. Kapphan, and E. Krätzig, "Hydrogen as origin of thermal fixing in $\mathrm{LiNbO}_{3}: \mathrm{Fe}$," Solid State Commun. 40, 543-545 (1981).

7. S. Klauer, M. Wöhlecke, and S. Kapphan, "Isotopic effect protonic conductivity in $\mathrm{LiNbO}_{3}$," Radiat. Effects Defects Solids 119, 699-704 (1991).

8. W. Meyer, P. Würfel, R. Munser, and G. Müller-Vogt, "Kinetics of fixation of phase holograms in $\mathrm{LiNbO}_{3}$," Phys. Status Solidi A 53, 171-180 (1979).

9. P. Hertel, K. H. Ringhofer, and R. Sommerfeldt, "Theory of thermal hologram fixing and application to $\mathrm{LiNbO}_{3}: \mathrm{Cu}$," Phys. Status Solidi A 104, 855-862 (1987).

10. M. Carrascosa and F. Agullo-Lopez, "Theoretical modeling of the fixing and developing of holographic gratings in $\mathrm{LiNbO}_{3}$," J. Opt. Soc. Am. B 7, 2317-2322 (1990).

11. A. Yariv, S. Orlov, G. Rakuljic, and V. Leyva, "Holographic fixing, readout, and storage dynamics in photorefractive materials," Opt. Lett. 20, 1334-1336 (1995).

12. N. V. Kukhtarev, V. B. Markov, S. G. Odulov, M. S. Soskin, and V. L. Vinetskii, "Holographic storage electrooptic crystals. I. Steady state," Ferroelectrics 22, 949-960 (1979). 
13. D. L. Staebler, W. J. Burke, W. Phillips, and J. J. Amodei, "Multiple storage and erasure of fixed holograms in Fe-doped $\mathrm{LiNbO}_{3}$," Appl. Phys. Lett. 26, 182-184 (1975).

14. J. F. Heanue, M. C. Bashaw, A. J. Daiber, R. Snyder, and L. Hesselink, "Digital holographic storage system incorporating thermal fixing in lithium niobate," Opt. Lett. 21, 1615-1617 (1996).

15. X. An and D. Psaltis, "Thermal fixing of 10,000 holograms in $\mathrm{LiNbO}_{3}: \mathrm{Fe}$," paper presented at the Optical Society of America Annual Meeting, Rochester, New York, 10-24 October 1996, paper MAAA5.

16. S. Orlov, "Holographic storage dynamics, phase conjugation, and nonlinear optics in photorefractive materials," Ph.D. dissertation (California Institute of Technology, Pasadena, Calif., 1996).
17. G. Burr, X. An, D. Psaltis, and F. Mok, "Large-scale rapid access holographic memory," in Optical Data Storage '95, G. R. Knight, H. Ooki, and S. Tyan, eds., Proc. SPIE 2514, 363-371 (1995).

18. The authors, along with F. H. Mok, are preparing the following paper for publication: "Large-scale random-access holographic memory using $\mathrm{LiNbO}_{3}$ :Fe."

19. D. Psaltis, D. Brady, X. G. Gu, and S. Lin, "Holography in artificial neural networks," Nature 343, 325-330 (1990).

20. D. Psaltis, D. Brady, and K. Wagner, "Adaptive optical networks using photorefractive crystals," Appl. Opt. 27, 17521759 (1988).

21. G. W. Burr and D. Psaltis, "Effect of the oxidation-state of $\mathrm{LiNbO}_{3}: \mathrm{Fe}$ on the diffraction efficiency of multiple holograms," Opt. Lett. 21, 893-895 (1996). 